\title{
Gatifloxacin Mesylate
}

National Cancer Institute

\section{Source}

National Cancer Institute. Gatifloxacin Mesylate. NCI Thesaurus. Code C132281.

The mesylate salt form of gatifloxacin, a synthetic 8-methoxyfluoroquinolone with antibacterial activity against a wide range of gram-negative and gram-positive microorganisms. Gatifloxacin exerts its effect through inhibition of DNA gyrase, an enzyme involved in DNA replication, transcription and repair, and inhibition of topoisomerase IV, an enzyme involved in partitioning of chromosomal DNA during bacterial cell division. 